\title{
A note of thanks
}

Solaf Elsayed ${ }^{*}$

At the end of this remarkable year, I wish to express my sincere gratitude, also on behalf of the other editors, the members of the editorial board and the publisher of the Egyptian Journal of Medical Human Genetics, to the referees of the articles considered for the 2021 volume of EJMHG. Hundreds of busy colleagues have spent their time in assessing close to 301 manuscripts submitted to our Journal, up from around 216 in 2020, in selecting the ones that may possibly be published, and in improving the quality of the selected articles. Many of the reviewers have helped us in the past years as well, and we are tremendously appreciative and grateful to them. It is largely thanks to them that EJMHG is doing well in attracting and publishing valuable exceptional articles in aspects of the genetic basis of human disease, with a particular emphasis on biochemical genetics, biological studies of enzymes and other protein deficiencies, as well as immunogenetics.

\section{Editor-in-Chief}

Solaf Elsayed, Ain-Shams University, Egypt

\section{Associate Editors}

Heba Elsedfy, Ain-Shams University, Egypt

Tanmoy Paul, D. N. C. College, Murshidabad, West Bengal, India

Mohd Adzim Khalili Bin Rohin, Universiti Sultan Zainal Abidin (UniSZA), Malaysia

Juan Carlos Balandrán, PhD. Laboratorio Juárez, Oaxaca, México

Darlen Cardoso de Carvalho, Doutora em Genetica e Biologia Molecular-UFPA, Brazil
RISHEE K. KALARIA, In-charge Head Bioinformatics Section, Aspee Shakilam Biotechnology Institute, Navsari Agricultural University, India

Naseem Akhter, Department of Laboratory Medicine, Faculty of Applied Medicine, Albaha University, Saudi Arabia

Heriberto Torres Moreno, Departamento de Ciencias Químico Biológicas y Agropecuarias, Universidad de Sonora, Unidad Regional Norte, Mexico

Amel Abdel Magied Elfaramawy, Prof of pediatrics and pediatric hepatology, faculty of medicine, Ain shams University, Egypt

Fatma Soliman Elsayed Ebeid, Professor of Pediatric Hematology Oncology and BMT, Ain Shams University, Egypt

Nermine Hussein Amr, Professor of Pediatrics, Ain Shams University, Egypt

Asmaa Wafeeq, consultant of child psychiatry, pediatrics department, Ain Shams University, Egypt

\section{Handling Editors}

Hosam Abdel-Ghany, Ain-Shams University, Egypt Naser Elhawary, Umm Al-Qura University Makkah, Saudi Arabia

Nermine Elsayed, Ain-Shams University, Egypt

Tarek Kamal, Ain-Shams University, Egypt

Sahar Nour El-Din, Ain-Shams University, Egypt

Asmaa Amrani, Ahmad Benbella University, Algeria

Lerine Bahyeldin Ahmad Elshazly, Head of Pediatric Hepatology Unit, Ain Shams University, Egypt

Rasha Aboelhassan, Naser Institute for research and treatment, Egypt

\footnotetext{
*Correspondence: elsayed683@yahoo.com
}

Ain-Shams University, Cairo, Egypt 


\section{Reviewers}

Gica Nicolae

Samir Abd El-Kaream

Aaser Abdelazim

Raafat Abdelfattah

Hosam Abdel-Ghany

Sayed Abdul Azeez

Shaimaa Abdulfattah

Rabab Abo Al-Kassem Ali

Rasha Aboelhassan

Ghalia Aboualchamat

Aly Aboulnasr

Salma Adham

Ali Ad'hiah

Ali Ad'hiah

Noha Adly

Younes Aftabi

Fahimeh Afzaljavan

Fatemeh Aghamahdi

Priyanka Ahimaz

Muzammil Ahmad Khan

Ahmed Farid E

Nejat Akar

Naseem Akhter

Enes Akyüz

Mahmood Al-Azzawi

Rebah Najah Algafari

Mohammad Al-Haggar

Abdullah S Alhomida

Fawad Ali

Imran Ali Khan

Azadeh Aliarab

Behnam Alipoor

Shahriar Alipour

Effat Alizadeh

Majd Aljamali

Ali Alkaissi

Risala Allami

Chaker Aloui

Abduldaem Alqasemi

Jorge L Alvarado-Socarras

Farshid Amiri

Asmaa Ammar

Nermine Amr

Asma Amrani-Midoun

Venkateshwari Ananthapur

Laura Antonaci

Yusha Araf

Salah Aref

Mahreen Arooj

Sana Ashiq

Maria Cristina Aspromonte

Emine İkbal Atlı
Azza Attia

Nikolay Avtandilyan

Diana S. Avzaletdinova

Duygu Aydemir

Sultan Aydın Köker

Kazi Faizul Azim

Swarnendu Bag

Gokhan Bagci

Dhan Raj Bagri

Gholamreza Bahari

Wael Bahia

Azza Baiomy

K Balakrishnan

Juan Carlos Balandrán

Claudia Banescu

Nikolay Barashkov

Arturo Becerra

Paulo Belmonte-de-Abreu

Zita Bendahan

Mounia Bendari

Anne Bergougnoux

Zahra Beyzaei

Deepa Bhat

Nadege Bondurand

J.Francis Borgio

Houssam Boulenouar

Nihan Bozkurt

Samantha Bruno

Metin Budak

Sowmya Gayatri C

Amanda Cambraia

Ayun Cassell

Filiz Basak Cengiz Ergin

Yusuf Ceylan

Abhijit Chakraborty

Divya Chandel

Nada Chaoul

Amrita Chaudhary

G.Rasul Chaudhry

Vikram Chauhan

Waseem Chauhan

Min-Hsin Chen

Massimiliano Chetta

Anna Cieslinska

Jean-Paul Concordet

Cenk Conkbayır

Mihaiela Cornea-Cipcigan

Maria Corrias

Ender Coskunpinar

Anna María Cueto-González

Sergio Cuevas-Covarrubias

Jelena Culej 
Marcus Fernando da Silva Praxedes

Marina Darenskaya

Ali Dawood

Mohammadreza Dehghani

Beltinge Demircioglu Kilıc

Debarati Dey

Maria Dobre

Guilherme Coutinho Kullmann Durate

Harsh Durga Tiwari

Vladimira Durmanova

Usha Dutta

Fatma Ebeid

Hisham Edinur

Maha Eid

Dalia Ezz El Din El Mikkawy

Heba Elabd

Mai Mohamed El-Daly

Mona Eldeeb

Eman Elghoroury

Marjanu Hikmah Elias

Jon Elkins

Sahar El-Masry

Manal Elsaid

Ayman Elsamanoudy

Amr Elsayed

Ghada Elsayed

Heba Elsedfy

Ayman El-Seedy

Ahmed El-Sohemy

Zafer ER

Hayriye Arzu Ergen

Ergen Arzu

Noha Esheba

Ahmet Emre Eşkazan

Nora Esmaiel

Reza Fadaei

Sultana Faradz

Pouremamali Farhad

Nairita Ahsan Faruqui

Botheina Farweez

Mohamed Fawzy

Alan Friedman

Seiji Fukuda

Linda Gailite

Doaa Gamal

Ibrahim Gehan

Maryam Gholamalizadeh

Bahari Gholamreza

Melissa Gilbert

Suzy Gohar

Gok Ilhami

Orgul Gokcen

Francianne Gomes Andrade
Jian-long Guan

Banu Guzel Nur

El-Bassyouni Hala T.

Mohammad Hamid

Biyan Nathanael Harapan

Anitha Haribalakrishna

Entesar Hassan

Atsushi Hattori

Ibrahim Haznedaroglu

Karen Heath

Gehan Hegazy

Katharina Helm

Iijima $\mathrm{Hi}$

Areerat Hnoonual

Salman Hosawi

Jeffery Hovis

Bo Huang

Liampas Ioannis

Alireza Isazadeh

Sagir Ismail

Marianne Issac

Janani Iyer

Golamreza Jadideslam

Leila Jahangiri

Ashish Jain

Chetan Jain

Anil Jalan

Hayarpi Javrushyan

Prabhash Jha

Sumi Elsa John

Amit Joshi

Wajih Kaabachi

Ahmed Kaftan

Rishee Kalaria

Stephan Kaler

Dean Kaličanin

Tarek Kamal

Azza Kamel

Ahmad Kantar

Juliana Tze-Wah Kao

Basudeba Kar

Kadri Karaer

Mohammad Karimian

Kaori Kimura-Kataoka

Nantarat Komanasin

Xiaomu Kong

Omid Kooshkaki

Nesrin Korkmaz

Kazumi Ktaguchi

Özlem Kucukhuseyin

Snehil Kumar

Vo Thi Thuong Lan

Jose Larios 
Lecke Sheila Bunecker

$\mathrm{Niu} \mathrm{Li}$

Zheqi Li

qiuxia Li

Lihua Li

Thomas Liehr

Chin-Yo Lin

Loannis Llias

Palanikumar Loganathan

Cristina Luongo

Sally M. Shalaby

Mashael S. Maashi

Debora Macis

Maryam Mahjoubin-Tehran

Simone Maistro

Sajid Malik

Jianping Mao

Matic Marija

Irena Marjanovic

Gerard Marshall Raj

Vladimir Maximov

Parvin Mehdipour

Beksac Mehmet Sinan

Wen Meng

Samia Menif

Diana Miclea

Poling Mikaela

Anamika Misra

Kinnari N. Mistry

Farnaz Mohajertehran

Sahar Mohamed

Ahmed Mohamed

Safarinejad Mohammad Reza

Taheri Mohsen

Majid Momeny

Alex France Messias Monteiro

Sebastian Morales-Pison

Magdy Mostafa

Somaia Mousa

Heba Moussa

Mrozikiewicz-Rakowska Beata

Werner Muller

Rosdiana Mus

Mohammed Mustak

Karkucak Mutlu

Shizuko Nagao

Arunasalam Naguleswaran

Jihon Nah

Rohini R. Nair

Martínez-Rodríguez Nancy

Prema Narayan

Seema Nayak

Gaber O. Moustafa
O'Donnell Jake S

Sabitu Olasupo

Jose Oliveira

Mônica Ghislaine Oliveira Alves

Linus Olson

Wael Osman

Tang Oushan

Venkatachalam Deepa Parvathi

Gang Peng

Maria pera

José Perdomo

Anna Pinto

Martin Poot

Nasser Pouladi

Rirturaj Purohit

Eman Rabie

Misbahuddin M Rafeeq

Zohreh Rahimi

Md Rezanur rahman

Christian Ramos-Peñafiel

Shahzada Mudasir Rashid

Stefano Ratti

Razi Bahman

Arnold J. J. Reuser

Maegan Roberts

Begum Rokeya

Eileen Roulis

Gianluca Russo

Mostafa Saadat

Alia Saeed

Mitali Sahni

Mortez Saki

Nevin Salah

Omneya Saleh

Hassanein Sally I.

Samir N.P. Kelada Samir N.P. Kelada

Made Saraswati

Pranabesh Sarkar

Bishajit Sarkar

Mohammad Sayyadi

Ali Sazci

Sahin, Sedef

Hesham Seif

Mohammad Seleem

N.V. semenova

Ikram Sghaier

Jay Shah

Farah Shahid

Gideon Shallangwa

Chaitra Shankar

John Shaughnessy

Anya Shindler

Vasily Shuvaev 
Jaqueline De Azevêdo Silva

Anja Hviid Simonsen

Sandeep Singh

Martina Skopkova

Jennifer Sloan

David Smith

Terry Smith

Abdel-Hady Soliman

Bruna Sorroche

Gordana Sosic

Paulo Roberto Souza

Abu Raghavan Srinivasan

Siti Aishah Sulaiman

Delfien Syx

Jinjing Tan

Daniela Tavian

Villarreal-Molina Teresa

Mukesh Thakur

Thilo Dörk-Bousset Thilo Dörk-Bousset

Eduardo Tizzano

Heriberto Torres Moreno

Jorg Tost

Md Sahab Uddin

Zulvikar Ulhaq

Umayal Branavan Umayal Branavan

Alexandra Usuga

Matilde Valeria Ursini

Lukas Varga

Anjo Veerman

Amit Kumar Verma

Ezzat Wafaa M.

Mohd Wamique

shuaishuai wang

Dao Wang

Pierre Warnault

David Weaver

Yongjun Wei

James Wiley

James Williams

Troy Wood

Sohier Yahia

Yu-Shun Yang

Osman Yokus

Zhang Yonggang

Yan Yousheng

Xiaotian Yuan

Yongyuth Yuthavong

Yves Ménézo Yves Ménézo

Viktoria V. Zabnenkova

Zakaria Zainab

Zangeneh Farideh Zafari

Jia Zeng

Lichao Zhang
Quan Zhang

Su Zhiguang Zhiguang

Rossokha Zoia

Published online: 28 December 2021

\section{Publisher's Note}

Springer Nature remains neutral with regard to jurisdictional claims in published maps and institutional affiliations.

\section{Submit your manuscript to a SpringerOpen ${ }^{\mathcal{O}}$} journal and benefit from:

- Convenient online submission

- Rigorous peer review

- Open access: articles freely available online

- High visibility within the field

- Retaining the copyright to your article

Submit your next manuscript at $\boldsymbol{\nabla}$ springeropen.com 